\title{
Coexpression of CMTM6 and PD-L1 as a predictor of poor prognosis in macrotrabecular-massive hepatocellular carcinoma
}

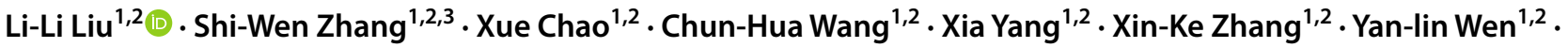 \\ Jing-Ping Yun ${ }^{1,2} \cdot$ Rong-Zhen Luo ${ }^{1,2}$
}

Received: 11 May 2020 / Accepted: 31 July 2020 / Published online: 7 August 2020

(c) The Author(s) 2020

\begin{abstract}
The "macrotrabecular-massive" (MTM) pattern of hepatocellular carcinoma (HCC) has been suggested to represent a distinct HCC subtype and is associated with specific molecular features. Since the immune microenvironment is heterogenous in HCC, it is important to evaluate the immune microenvironment of this novel variant. CMTM6, a key regulator of PD-L1, is an important immunocheckpoint inhibitor. This study aimed to evaluate the prognostic effect of CMTM6/PD-L1 coexpression and its relationship with inflammatory cells in HCC. We analyzed 619 HCC patients and tumors were classified into MTM and non-MTM HCC subtypes. The expression levels of CMTM6 and PD-L1 in tumor and inflammatory cells were evaluated by immunohistochemistry. The density of inflammatory cells in the cancer cell nest was calculated. Tumoral PD-L1 expression and inflammatory cell density were higher in the MTM type than in the non-MTM type. CMTM6-high expression was significantly associated with shorter OS and DFS than CMTM6-low expression in the whole HCC patient population and the MTM HCC patient population. Moreover, MTM HCC patients with CMTM6/PD-L1 coexpression experienced a higher risk of HCC progression and death. In addition, CMTM6/PD-L1 coexpression was shown to be related to a high density of inflammatory cells. Notably, a new immune classification, based on CMTM6/PD-L1 coexpression and inflammatory cells, successfully stratified OS and DFS in MTM HCC. CMTM6/PD-L1 coexpression has an adverse effect on the prognosis of HCC patients, especially MTM HCC patients. Our study provides evidence for the combination of immune status assessment with anti-CMTM6 and anti-PD-L1 therapy in MTM HCC patients.
\end{abstract}

Keywords CMTM6 · PD-L1 · Inflammatory cells $\cdot$ HCC $\cdot$ Prognosis

Li-Li Liu, Shi-Wen Zhang and Xue Chao have contributed equally to this work.

Electronic supplementary material The online version of this article (https://doi.org/10.1007/s00262-020-02691-9) contains supplementary material, which is available to authorized users.

Rong-Zhen Luo

luorzh@sysucc.org.cn

1 State Key Laboratory of Oncology in South China, Collaborative Innovation Center for Cancer Medicine, Sun Yat-Sen University Cancer Center, Guangzhou 510060, China

2 Department of Pathology, Sun Yat-Sen University Cancer Center, 651\# Dong Feng Road East, Guangzhou 510060, Guangdong, China

3 Department of Pathology, The Eighth Affiliated Hospital, Sun Yat-Sen University, Shenzhen 51800, China

\section{Introduction}

Malignant primary liver tumors are the second leading cause of cancer-related death worldwide, with an increasing incidence in almost all countries [1]. Hepatocellular carcinoma (HCC) is about $85-90 \%$ of primary liver cancer and its main risk factors are hepatitis $\mathrm{B}(\mathrm{HBV})$ or $\mathrm{C}(\mathrm{HCV})$ infection, alcohol consumption and metabolic syndrome [2,3]. Early HCC can be treated by local ablation, surgical resection or liver transplantation. Kinase and immunocheckpoint inhibitors have been shown to be effective options for the treatment of advanced HCC [4]. Among HCC subtypes, the trabecular pattern is the most common growth pattern of HCC and mimics normal hepatic cord plates. When the trabeculae become $>6$ cells thick, the growth pattern is referred to as "macrotrabecular-massive" (MTM), and when it is $>50 \%$ of the entire tumor, it is considered a subtype: MTM-HCC $[5,6]$. Recent studies have shown that MTM-HCC has an 
aggressive phenotype and distinct biological (high alpha-feto protein serum levels) and molecular (G3 transcriptomic subgroup, TP53 mutations and FGF19 amplifications) features [5]. There is a critical clinical need to explore the immune microenvironment of this novel HCC subtype.

Blockade of the PD-L1/PD-1 interaction with monoclonal antibodies represents a milestone for anticancer immunotherapy. Indeed, agents targeting PD-1/PD-L1 were recently reported to induce impressive antitumor effects in HCC [7]. Patients with positive PD-L1 expression had significantly poorer DFS and OS than PD-L1 negative patients [8]. Blocking CSF1/CSFR1 prevents tumor-associated macrophage trafficking and is also associated with high responsiveness to PD-1 blockade [9]. Notably, intratumoral heterogeneity of PD-L1 expression has been frequently observed in HCC [10]. Tumors not expressing detectable levels of PD-L1 can also respond to PD- 1 inhibitors. Therefore, another predictor to supplement PD-L1 is needed.

CMTM6 belongs to the chemokine-like factor (CKLF)like MARVEL transmembrane domain-containing (CMTM) family and is broadly expressed at the plasma membrane of various cells, but the biological function of this ubiquitously expressed protein was unknown until recently [11]. MARVEL domain proteins have been suggested as key regulators of PD-L1 in tumor cells [12]. CMTM6 promotes PD-L1 expression in tumor cells in the defense against $\mathrm{T}$ cells [13]. In contrast, the depletion of CMTM6 relieves T cell immunosuppression [14]. Additionally, it has been reported that elevated CMTM6 in head and neck squamous cell carcinoma and glioma is associated with a poor prognosis [12, 15], and a potential therapeutic target for renal clear cell carcinoma [16]. CMTM6 expression in combination with PD-L1 expression can be used as a prognostic and therapeutic indicator in lung cancer and pancreatic ductal adenocarcinoma [17-20]. Previous studies found that CMTM6 was downregulated in HCC tissues and correlated with HCC metastasis and survival in HCC patients, the polymorphisms of rs164207 in CMTM6 was found in HCC [21-23]. In addition, CMTM6 showed decreased expression in nonneoplastic liver cells after tumor promotion with piperonyl butoxide (PBO) in mice [24]. These findings suggest the potential value of CMTM6 as a therapeutic target. However, the association between CMTM6 and the immune microenvironment has not been evaluated in HCC subtypes.

In our study, we explored the relationship between CMTM6 expression, clinicopathological variables, and the immune microenvironment by analyzing data from the whole population and two histological subtypes. Then, based on CMTM6 expression, PD-L1 expression and inflammatory cell density, we optimized the present immune classification and established a novel immunophenotyping system in HCC, especially in MTM-HCC. Our study may provide evidence for HCC patients to choose proper immunotherapy.

\section{Materials and methods}

\section{Patients and samples}

Our study was approved by the Institutional Ethical Boards of Sun Yat-sen University Cancer Center. Patient data were retrospectively analyzed from two subtypes: the MTM type (316 patients) and the non-MTM type (303 patients). Patients in both subtypes underwent surgical resection for HCC from Jan. 2000 to Dec. 2010. Informed consent was obtained from all patients. The mean follow-up time was 32.3 months, and the sample included 549 (88.7\%) males and $70(11.3 \%)$ females. The mean age was 49 years, ranging from 13 to 77 years.

\section{Tissue microarray (TMA) construction}

HCC tissues and adjacent nontumorous hepatic tissue samples were collected and constructed for TMA. Primary antibodies (anti-CMTM6: Sigma-Aldrich, HPA 026980; anti-PD-L1: Roche, SP263) were incubated at $4{ }^{\circ} \mathrm{C}$, washed three times with phosphate-buffered saline, incubated with biotinylated goat anti-mouse antibodies, and then stained with DAKO liquid 3,3'-diaminobenzidine tetrahydrochloride (DAB) and finally with Mayer's hematoxylin. TMA slides stained with CMTM6 and PD-L1 were observed under a microscope, and the protein expression levels of CMTM6 and PD-L1 were assessed by two independent pathologists (Shi-Wen Zhang and Xue Chao).

The CMTM6 positively stained samples were scored as follows: 0, less than 5\% positively stained cells; $1,6-19 \%$ positively stained cells; $2,20-49 \%$ positively stained cells; $3,50-74 \%$ positively stained cells; $4,75-100 \%$ positively stained cells. The intensity was scored as follows: 0 , negative staining; 1 , weak staining; 2 , moderate staining; and 3 , strong staining. The final score was calculated by multiplying the percentage score by the staining intensity score. The median IHC score of 3 was chosen as the cut-off value for defining high and low CMTM6 expression. For tumor PD-L1 expression, the percentages of cells demonstrating membranous staining for PD-L1 among total tumor cells were quantified, consistent with previous studies [25, 26]. For inflammatory cell PD-L1 expression, any expression $(\geq 1 \%)$ of PD-L1 on tumor infiltrating and stromal immune cells was considered present. The tumor and inflammatory cell PD-L1 positivity threshold was defined as at least $1 \%$ displaying membranous PD-L1 staining of any intensity. The density of inflammatory cells was manually counted in five separate fields under $\times 200$ high-power magnification. The inflammatory cell positivity threshold was defined as at least $1 / \mathrm{mm}^{2}$. Quantification was conducted independently by two experienced pathologists who were blinded to the 
clinical data of patients, any discrepancies in scoring were adjudicated.

\section{Statistical analysis}

SPSS 19.0 was used to perform statistical analyses (SPSS, Chicago, IL, USA). Student's $t$ test was used to assess the significance of differences in CMTM6 and PD-L1 expression and inflammatory cell density levels. The Chi-square test was used to analyze the correlation between CMTM6 expression and clinicopathological parameters in HCC patients. Pearson's $\chi 2$ test was used to analyze the correlation between the expression of CMTM6 and PD-L1. OS and DFS were analyzed by Kaplan-Meier analysis and compared by log-rank test. Univariate and multivariate Cox regression analyses were used to analyze prognostic correlations. $P<0.05$ was considered statistically significant.

\section{Results}

\section{Patient baseline characteristics according to tumor histological subtype}

A total of $619 \mathrm{HCC}$ patients who underwent primary tumor resection were analyzed in this study. The clinicopathological variables of patients with the two histological subtypes of HCC are listed in Supplementary Table 1. Representative IHC images for CMTM6 and PD-L1 in HCC are shown in Figs. 1 and 2. In both histological subtypes, the expression rates of CMTM6 expression were high, at 63.9\% (202/316) and $52.8 \%$ (160/303); interestingly enough, these rates were significantly higher than the rates of tumoral PD-L1 expression, which were $43.0 \%$ (136/316) and 34.0\% (103/303) (Supplementary Table 1). Consistent with previous studies, our results showed that MTM type has a worse prognosis than non-MTM type (Supplementary Fig. 1a, b).

\section{PD-L1 expression, inflammatory cell density and CMTM6 expression in HCC tissues}

We examined PD-L1 expression in tumor tissues and found that $38.6 \%$ (239/619) of patients were positive for tumoral PD-L1, 50.1\% (310/619) of patients were positive for inflammatory cell PD-L1 and 2.4\% (15/619) of patients were positive for adjacent normal cell PD-L1 (Fig. 1a; Supplementary Table 1). The percentage of PD-L1+tumor cells was higher in MTM-type tumors than in non-MTM type tumors (Fig. 1b). The density of inflammatory cells in the tumoral region was significantly higher in MTM type tumors than in non-MTM type tumors (Fig. 1c, d). However, inflammatory cell PD-L1 and normal cell PD-L1 expression was not significantly different between the MTM type and non-MTM type (Fig. 1b). Representative photomicrographs are shown in Fig. 1e.

We next further confirmed the expression profile of CMTM6 in HCC. Representative IHC images of CMTM6 expression are shown in Fig. 2a. CMTM6 expression in HCC was significantly higher than that in nontumorous tissues (Fig. 2b, c). The expression of CMTM6 was significantly higher in MTM-type tumors than in non-MTM-type tumors (Fig. 2d, e). In another TMA cohort consisting of 47 HCC cases with portal vein embolus, CMTM6 expression was not significantly different between primary lesions and tumor embolus metastases (Supplementary Fig. 1c, d).

\section{Relationship of CMTM6 and PD-L1 expression and clinicopathological features}

The association between CMTM6 expression and clinicopathological characteristics is shown in Table 1. CMTM6 expression was significantly correlated with high AFP level $(P=0.032)$, tumor size $(P=0.016)$, advanced TNM stage $(P<0.001)$, vascular invasion $(P=0.001)$, and lymph node metastasis $(P=0.030)$ in the MTM type but not in the nonMTM type. This significant association was also detected between CMTM6 and tumoral PD-L1 expression in both subtypes (MTM type: $P<0.001$; non-MTM type: $P=0.004$ ).

The association between tumoral PD-L1 expression and clinicopathological variables is shown in Supplementary Table 2. Tumor PD-L1 positivity was significantly correlated with age $(P=0.032)$, HBV positivity $(P=0.045)$, and high AFP level $(P=0.008)$ in the MTM type but not in the nonMTM type. A significant association was identified between tumoral PD-L1 positivity and high inflammatory cell density in both subtypes (MTM type: $P<0.001$; non-MTM type: $P<0.001)$.

\section{Relationship between CMTM6/PD-L1 coexpression and inflammatory cells in HCC}

We also detected the density of inflammatory cells in HCC. Representative figures are shown in Fig. 3a. An association was found between high CMTM6 expression and tumoral PD-L1 in the whole population $\left(R^{2}=0.006, P=0.048\right)$ and in MTM-type HCC $\left(R^{2}=0.006, P=0.050\right)$, but no significant association was found in non-MTM-type HCC (Fig. 3b). A positive association was detected between high CMTM6 expression and inflammatory cell PD-L1 in the whole population $(P=0.006)$ and MTM type $(P=0.027)$ (Fig. 3c). As shown in Fig. 3d, no significant difference was found between CMTM6-high and CMTM6-low tumors. The density of inflammatory cells in the PD-L1+ tumors was significantly higher than that in PD-L1- tumors in the whole population $(P=0.021)$. Furthermore, the density of inflammatory cells was highest in group III (CMTM6-high/ 
A

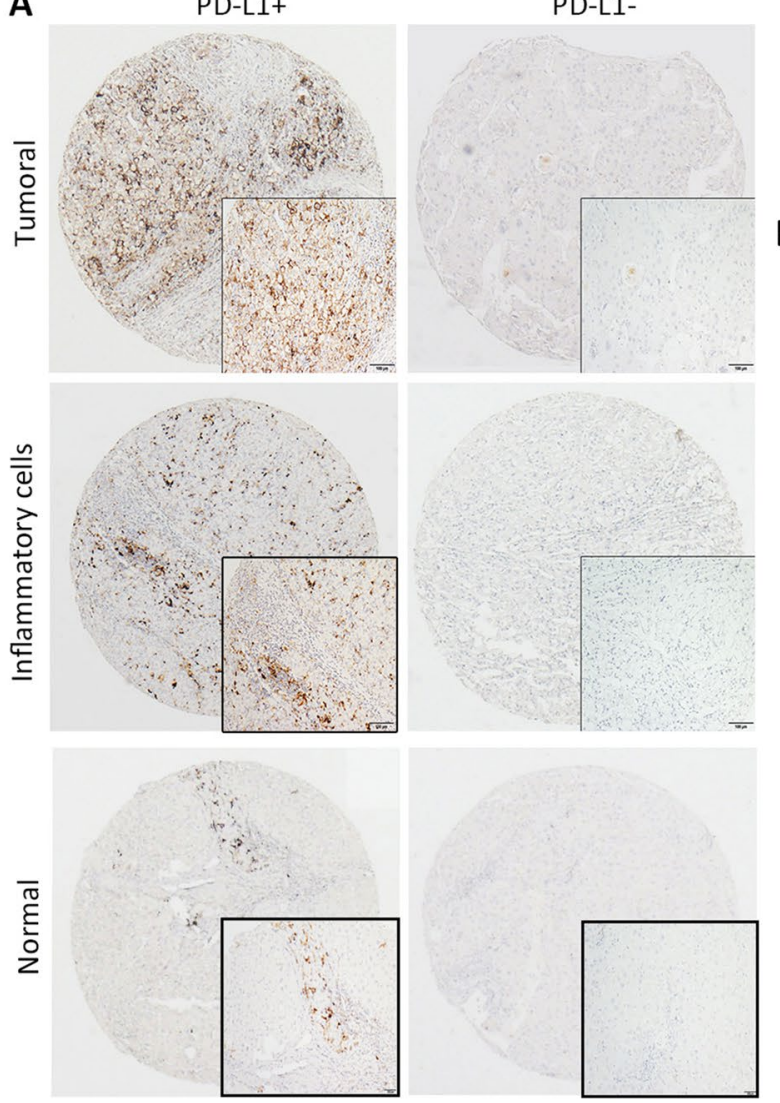

C
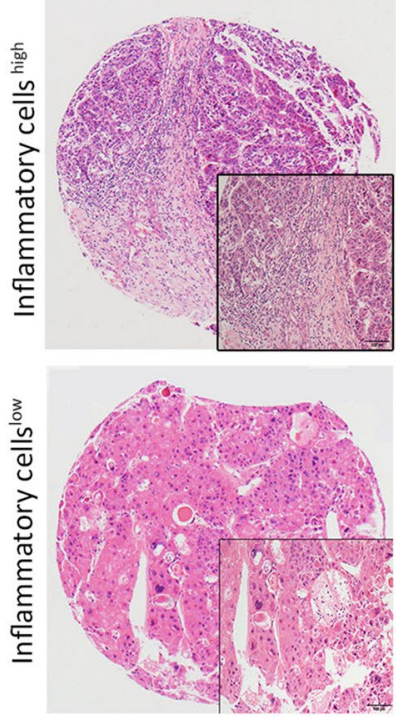

B

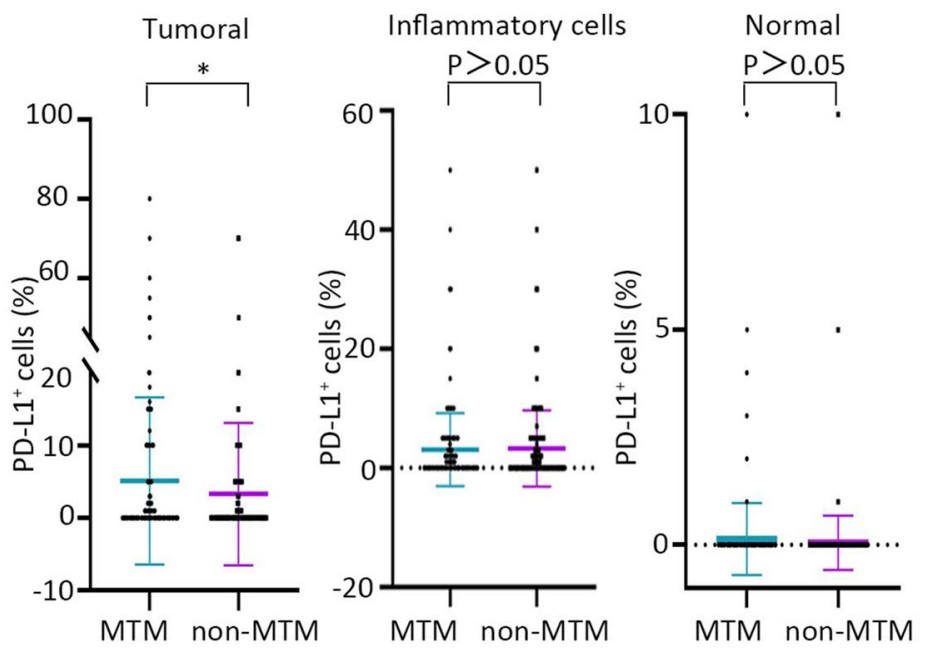

D

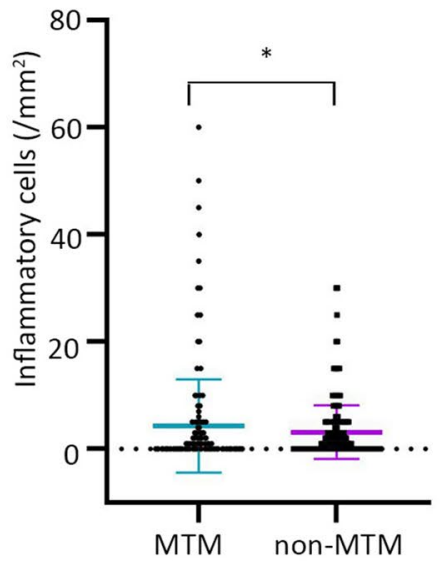

$\mathbf{E}$
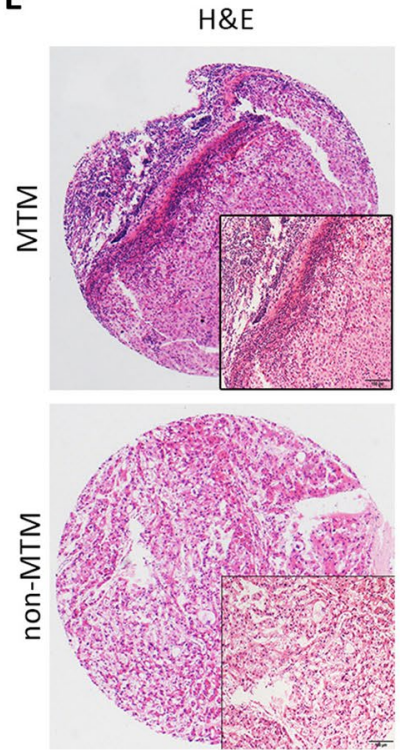

PDL1
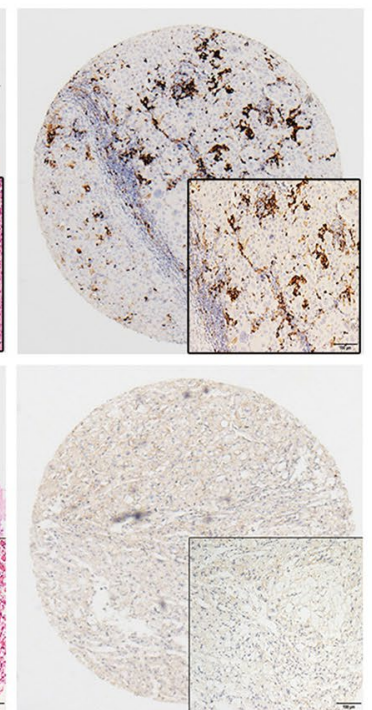

Fig. 1 PD-L1 expression and inflammatory cell density in MTM subtype and non-MTM subtype of HCC. a Expression of PD-L1 in HCC detected by IHC. Tumoral PD-L1-positive and -negative tumors (upper panel), inflammatory cell PD-L1-positive and -negative tumors (middle panel) and adjacent normal cell PD-L1-positive and -negative expression (lower panel). b Dot plots of tumoral, inflammatory cell and normal cell PD-L1-positive percentage in MTM type vs. non-MTM type tumors. $P$ values were calculated using the Mann-
Whitney $U$ test. c Representative images of high and low inflammatory cell density in MTM type and non-MTM type tumors. d Dot plot of inflammatory cells in MTM type vs. non-MTM type tumors. e Representative HE and IHC images of high inflammatory cells density and tumoral PD-L1-positve in MTM type (upper panel) and low inflammatory cell density and tumoral PD-L1-negative in non-MTM type (lower panel). Quantitative data are presented as mean \pm SD 
PD-L1+) in the whole population $(P=0.045)$. In MTM type and non-MTM type, no significant difference among different subgroup were detected (Supplementary Fig. 1e, f).

\section{Impact of CMTM6 and PD-L1 expression on the overall survival and disease-free survival of HCC patients}

To explore the prognostic significance of CMTM6 and PD-L1, Cox proportional hazards regression was performed. Among the whole population, CMTM6-high patients had significantly increased risks of disease progression and all causes of death compared with CMTM6-low patients (OS: HR $=1.493, P<0.001$; DFS: HR $=1.369, P=0.010$ ) and MTM type patients (OS: HR $=1.574, P<0.001$; DFS: $\mathrm{HR}=1.624, P=0.008)$. In the non-MTM type, patients with high CMTM6 tended to have a poor prognosis (OS: $\mathrm{HR}=1.310, P=0.035)$, but there was no association with DFS (Table 2; Supplementary Table 3).

Kaplan-Meier analysis revealed that CMTM6 expression was significantly associated with worse DFS and OS in the whole population (OS: $P<0.001$; DFS: $P=0.010)$ and in the MTM type (OS: $P<0.001$; DFS: $P=0.008$ ) but not in the non-MTM type (Fig. 4a; Supplementary Fig. 2a). Stratified survival analysis further confirmed the prognostic value of CMTM6 (Supplementary Fig. 3; Table 1). Multivariate analysis indicated that high CMTM6 expression was an independent prognostic marker for HCC in whole population $(\mathrm{HR}=1.056, P=0.030)$, but not in the MTM type and non-MTM type (Table 3).

Similar to the patterns observed with CMTM6, tumoral PD-L1+ patients suffered much higher mortality rates than PD-L1 - patients in the whole population and the MTM type $(\mathrm{HR}=1.217, P=0.029$ in the whole population; $\mathrm{HR}=1.610$, $P<0.001$ in the MTM type) but not in the non-MTM type population (Table 2). Kaplan-Meier analysis also suggested that PD-L1 positivity was significantly associated with worse OS in the whole population and the MTM type (Fig. 4b, whole population, $P=0.028$; MTM type, $P<0.001$ ) but was not associated with worse OS in the non-MTM type. PD-L1 expression was not significantly associated with worse DFS in the respective groups (Supplementary Fig. 2b).

\section{Coexpression of CMTM6 and PD-L1 in HCC and prognostic significance}

Considering that CMTM6 has a regulator function on PD-L1, we attempted to explore the prognostic impact of CMTM6/PD-L1 coexpression in HCC. The proportions of patients with CMTM6-high/PD-L1+, CMTM6-high/ PD-L1 - or CMTM6-low/PD-L1+, and CMTM6-low/ PD-L1 - expression are shown in Supplementary Table 1. Patients were divided into three groups: group I, both negative (CMTM6-high/PD-L1-); group II, single positive (CMTM6-high/PD-L1- or CMTM6-high/PD-L1+; and group III, both positive (CMTM6-high/PD-L1+).

Kaplan-Meier analysis demonstrated that both OS and DFS in group III were significantly reduced compared with those in group I and group II in the whole population and those in the MTM type, but not in the non-MTM type (Fig. 4c; Supplementary Fig. 2c). Importantly, multivariate analysis revealed that CMTM6-high/PD-L1+ still had a significant impact on $\mathrm{OS}$ in the whole population $(\mathrm{HR}=1.213$, $P=0.005)$ and MTM type $(\mathrm{HR}=1.297, P=0.003)$ but not in the non-MTM type (Supplementary Table 4).

\section{Immune classification for HCC}

We classified the patients into six types based on inflammatory cell and CMTM6/PD-L1 coexpression to provide rationale for immunotherapy. As shown in Fig. $4 d$ and Supplementary Fig. 2d, in the inflammatory cell positive subgroups, Kaplan-Meier analysis demonstrated that both OS and DFS in type BIII were significantly reduced compared with type BI and type BII in the whole population and the MTM type, but there was no significant difference in the non-MTM type. Furthermore, in the inflammatory cell-negative subgroup, OS in type AIII was significantly reduced compared with that in type AI and type AII in the whole population and the MTM type, but this difference was not observed in the non-MTM type (Supplementary Fig. 1g). However, no significant difference in DFS was detected among the three groups (Supplementary Fig. 2e).

\section{Discussion}

The immunological properties of human malignancies can vary greatly according to tumor origin and histological type and often display diverse immune cell recruitment. This study is the first to reveal remarkable differences in CMTM6 and PD-L1 expression and inflammatory cell density between the two subtypes of HCC, which are intimately related to their tumor biology and clinical outcomes. We found significantly high tumoral PD-L1 expression, high CMTM6 expression and high inflammatory cell density in the MTM type, whereas the non-MTM type showed low inflammatory cell density. CMTM6/PD-L1 expression was an independent prognostic factor for patient survival in the whole population and the MTM subtype population, confirming the crucial roles of these markers in the pathogenesis of HCC. In addition, there was a significant association between CMTM6 and tumoral PD-L1 expression in these two subtypes but no significant association between CMTM6 and inflammatory cell PD-L1 expression in nonMTM subtypes, suggesting that high CMTM6 activity in 

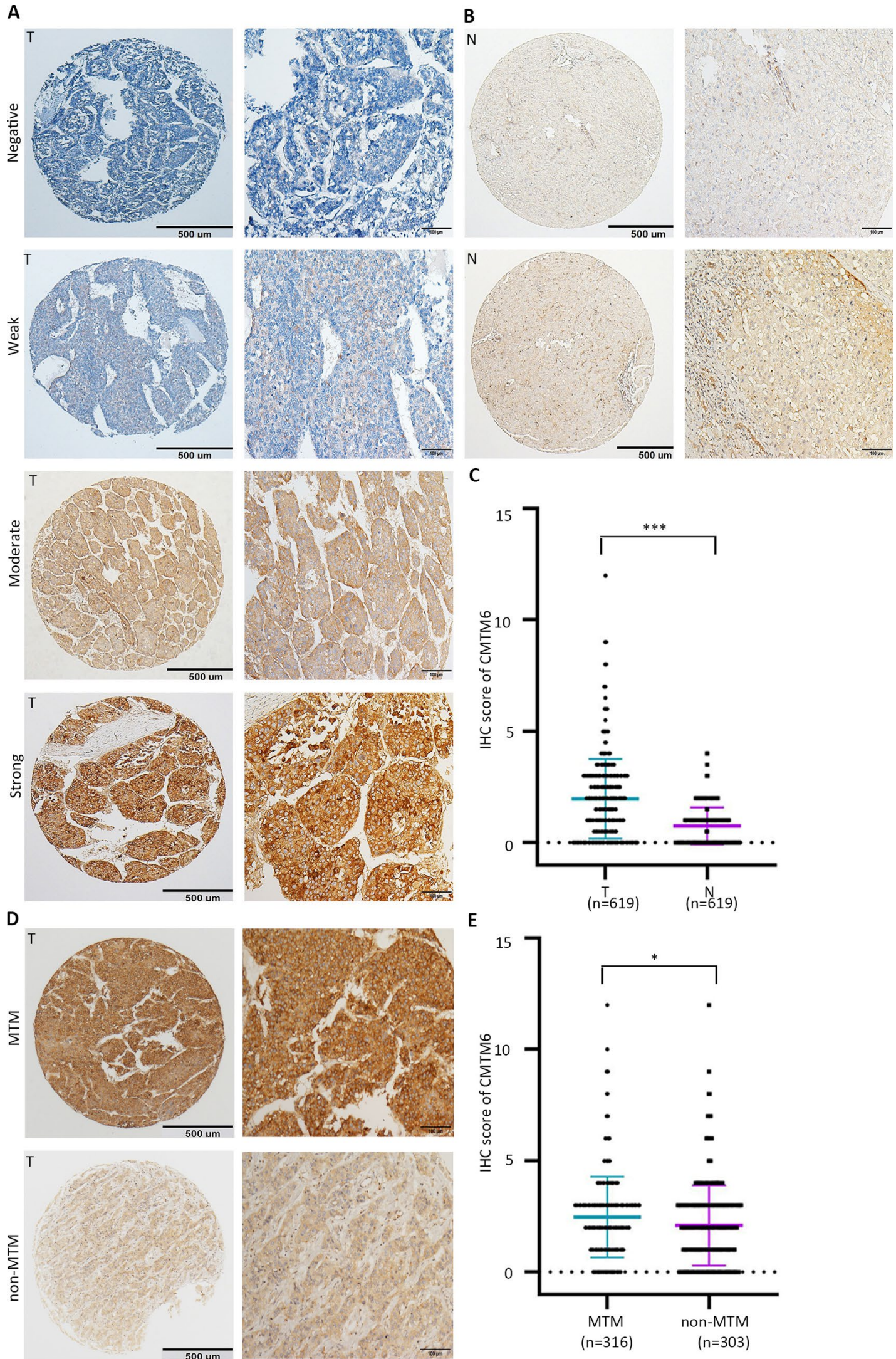
4Fig. 2 Overexpression of CMTM6 in HCC detected by IHC. a Representative images of IHC staining for CMTM6 expression in a TMA cohort. Representative images of negative, weak, moderate and strong (top to bottom) density staining for tumor tissues are shown. b Representative IHC images of negative (top) and positive (bottom) in nontumor tissues are presented. c The IHC scores of the TMA cohort, including 619 HCC patients. $P$ values were calculated using the Student's $t$ test. d Representative images of weak and strong density staining in MTM type and non-MTM type tumors. e Dot plots of IHC score in MTM type vs. non-MTM type tumors. Quantitative data are presented as mean $\pm \mathrm{SD}$
HCC contributes to tumoral PD-L1 expression. Collectively, these findings provide an important clue for deciphering the distinct clinical features of the two subtypes in patients with HCC.

Recent studies have shown that CMTM6 combined with its family member CMTM4 can maintain the stability of PD-L1 and prevent PD-L1 from lysosome hydrolyzation in multiple tumor types, such as melanoma, breast cancer, and lung cancer $[13,14]$. Zhu et al. showed that CMTM6

Table 1 The association of clinicopathological parameters with CMTM6 expression in the MTM and non-MTM HCC

\begin{tabular}{|c|c|c|c|c|c|c|c|c|c|c|c|c|}
\hline \multirow[t]{3}{*}{ Variables } & \multicolumn{4}{|c|}{ Overall $(n=619)$} & \multicolumn{4}{|c|}{$\operatorname{MTM}(n=316)$} & \multicolumn{4}{|c|}{ Non-MTM $(n=303)$} \\
\hline & \multirow[t]{2}{*}{$n$} & \multicolumn{3}{|c|}{ CMTM6 expression } & \multirow[t]{2}{*}{$n$} & \multicolumn{3}{|c|}{ CMTM6 expression } & \multirow[t]{2}{*}{$n$} & \multicolumn{3}{|c|}{ CMTM6 expression } \\
\hline & & Low & High & $P$ value & & Low & High & $P$ value & & Low & High & $P$ value \\
\hline Age (years) ${ }^{\mathrm{a}}$ & & & & 0.722 & & & & 0.816 & & & & 0.230 \\
\hline$<50$ & 291 & 123 & 168 & & 166 & 65 & 101 & & 125 & 58 & 67 & \\
\hline$\geq 50$ & 328 & 134 & 194 & & 150 & 49 & 101 & & 178 & 85 & 93 & \\
\hline Gender & & & & 0.005 & & & & 0.188 & & & & 0.004 \\
\hline Male & 549 & 217 & 332 & & 273 & 90 & 183 & & 276 & 127 & 149 & \\
\hline Female & 70 & 40 & 30 & & 43 & 24 & 19 & & 27 & 16 & 11 & \\
\hline HBV & & & & 0.641 & & & & 0.950 & & & & 0.463 \\
\hline Positive & 47 & 18 & 29 & & 24 & 7 & 17 & & 23 & 11 & 12 & \\
\hline Negative & 572 & 239 & 333 & & 292 & 107 & 185 & & 280 & 132 & 148 & \\
\hline $\operatorname{AFP}(\mathrm{ng} / \mathrm{ml})$ & & & & 0.012 & & & & 0.032 & & & & 0.374 \\
\hline$<20$ & 149 & 75 & 74 & & 61 & 25 & 36 & & 88 & 50 & 38 & \\
\hline$\geq 20$ & 470 & 182 & 288 & & 255 & 89 & 166 & & 215 & 93 & 122 & \\
\hline Cirrhosis & & & & 0.557 & & & & 0.537 & & & & 0.939 \\
\hline Yes & 109 & 48 & 61 & & 52 & 19 & 33 & & 57 & 29 & 28 & \\
\hline No & 510 & 209 & 301 & & 264 & 95 & 169 & & 246 & 114 & 132 & \\
\hline Tumor multiplicity & & & & 0.087 & & & & 0.053 & & & & 0.758 \\
\hline Single & 427 & 187 & 240 & & 210 & 77 & 133 & & 217 & 110 & 107 & \\
\hline Multiple & 192 & 70 & 122 & & 106 & 37 & 69 & & 86 & 33 & 53 & \\
\hline Tumor size $^{\mathrm{b}}$ & & & & 0.003 & & & & 0.016 & & & & 0.181 \\
\hline$<5 \mathrm{~cm}$ & 168 & 86 & 82 & & 70 & 30 & 40 & & 98 & 56 & 42 & \\
\hline$\geq 5 \mathrm{~cm}$ & 451 & 171 & 280 & & 246 & 84 & 162 & & 205 & 87 & 118 & \\
\hline Differentiation & & & & 0.098 & & & & 0.111 & & & & 0.842 \\
\hline Well & 58 & 30 & 28 & & 21 & 8 & 13 & & 37 & 22 & 15 & \\
\hline Moderate-poor & 561 & 227 & 334 & & 295 & 106 & 189 & & 266 & 121 & 145 & \\
\hline TNM stage & & & & 0.001 & & & & 0.000 & & & & 0.567 \\
\hline I-II & 377 & 176 & 201 & & 179 & 67 & 112 & & 198 & 109 & 89 & \\
\hline III-IV & 242 & 81 & 161 & & 137 & 47 & 90 & & 105 & 34 & 71 & \\
\hline Vascular invasion & & & & 0.002 & & & & 0.001 & & & & 0.418 \\
\hline No & 521 & 230 & 291 & & 250 & 93 & 157 & & 271 & 137 & 134 & \\
\hline Yes & 98 & 27 & 71 & & 66 & 21 & 45 & & 32 & 6 & 26 & \\
\hline Tumor capsule & & & & 0.151 & & & & 0.358 & & & & 0.396 \\
\hline Complete & 351 & 137 & 214 & & 190 & 65 & 125 & & 161 & 72 & 89 & \\
\hline Incomplete & 268 & 120 & 148 & & 126 & 49 & 77 & & 142 & 71 & 71 & \\
\hline LN metastasis & & & & 0.226 & & & & 0.030 & & & & 0.653 \\
\hline No & 587 & 247 & 340 & & 299 & 107 & 192 & & 288 & 140 & 148 & \\
\hline Yes & 32 & 10 & 22 & & 17 & 7 & 10 & & 15 & 3 & 12 & \\
\hline Cytological type & & & & 0.111 & & & & 0.124 & & & & 0.447 \\
\hline Liver cell & 538 & 231 & 307 & & 277 & 102 & 175 & & 261 & 129 & 132 & \\
\hline
\end{tabular}


Table 1 (continued)

\begin{tabular}{|c|c|c|c|c|c|c|c|c|c|c|c|c|}
\hline \multirow[t]{3}{*}{ Variables } & \multicolumn{4}{|c|}{ Overall $(n=619)$} & \multicolumn{4}{|c|}{$\operatorname{MTM}(n=316)$} & \multicolumn{4}{|c|}{ Non-MTM $(n=303)$} \\
\hline & \multirow[t]{2}{*}{$n$} & \multicolumn{3}{|c|}{ CMTM6 expression } & \multirow[t]{2}{*}{$n$} & \multicolumn{3}{|c|}{ CMTM6 expression } & \multirow[t]{2}{*}{$n$} & \multicolumn{3}{|c|}{ CMTM6 expression } \\
\hline & & Low & High & $P$ value & & Low & High & $P$ value & & Low & High & $P$ value \\
\hline Clear cell & 40 & 16 & 24 & & 19 & 8 & 11 & & 21 & 8 & 13 & \\
\hline Fatty-rich & 33 & 9 & 24 & & 16 & 3 & 13 & & 17 & 6 & 11 & \\
\hline Giant cell & 8 & 1 & 7 & & 4 & 1 & 3 & & 4 & 0 & 4 & \\
\hline Tumoral PD-L1 & & & & 0.000 & & & & 0.000 & & & & 0.004 \\
\hline Negative & 380 & 188 & 192 & & 180 & 77 & 103 & & 200 & 111 & 89 & \\
\hline Positive & 239 & 69 & 170 & & 136 & 37 & 99 & & 103 & 32 & 71 & \\
\hline Inflammatory cell PD-L1 & & & & 0.000 & & & & 0.000 & & & & 0.093 \\
\hline Negative & 309 & 152 & 157 & & 152 & 62 & 90 & & 157 & 90 & 67 & \\
\hline Positive & 310 & 105 & 205 & & 164 & 52 & 112 & & 146 & 53 & 93 & \\
\hline Inflammatory cells & & & & 0.000 & & & & 0.012 & & & & 0.007 \\
\hline Low & 264 & 133 & 131 & & 124 & 56 & 68 & & 140 & 77 & 63 & \\
\hline High & 355 & 124 & 231 & & 192 & 58 & 134 & & 163 & 66 & 97 & \\
\hline
\end{tabular}

$A F P$ a-fetoprotein, $H B V$ hepatitis B virus infection, $L N$ lymph node ${ }^{*} P$ value $<0.05$ in bold are statistically significant

${ }^{\mathrm{a}}$ Median age; ${ }^{\mathrm{b}}$ Median tumor size

was downregulated in HCC tissues, supporting a tumor suppressive role of CMTM6 in HCC [21]. However, according to our data, CMTM6 expression was up-regulated in HCC tissues. Elevated expression of CMTM6 was frequently accompanied with worse malignant phenomenon, such as with high AFP level, large tumor size, advanced TNM stage, vascular invasion in a large cohort of 619 HCC cases (In Zhu's study, only 75 HCC samples were collected). Our data was in line with other studies indicating that increased CMTM6 expression was presented in gliomaand Head and Neck Squamous Cell Carcinoma, and CMTM6 positivity was associated with shorter DFS and OS [12, 15]. Collectively, we consider our data are more representative to show the expression of CMTM6 in HCC. Moreover, high expression of CMTM6 was an independent prognostic factor in the whole population. Expression of CMTM6 was more frequent than expression of PD-L1, consistent with prior literature $[18,19]$, which indicates that CMTM6 could be regarded as a potential target for immunotherapy. Consistent with our results, upregulation of PD-L1 is observed during HBV infection [27]. Recent studies have shown that extracellular vesicles (EVs) produced by HBV-infected hepatocytes are endocytosed by circulating monocytes resulting in PD-L1 upregulation [28, 29]. Interestingly, another study showed that serum soluble PD-L1 (sPD-L1) concentration was several-fold higher in HBV-related HCC than in healthy control, a significant difference, while sPDL1 was positively correlated with tumor PD-L1 expression [30]. During chronic HBV infection, virus particles are continuously released from virus-infected cells and maintain a network of immunosuppressive mechanisms that interfere with virus elimination [31]. That may explain the reason why PD-L1 expression correlated with HBV infection. We are also very interested in why age is related to PD-L1 expression. However, when we used the Student's $t$ test to analyze the difference in the mean age between the PD-L1 positive group and the negative group, we did not observe with a significant difference ( 48.62 years vs 50.38 years, $P=0.078$ ). It may need more data to support to concluded that PD-L1 is age-related.

Although the expression of both CMTM6 and PD-L1 is induced by related immunoregulatory factors, a previous report indicated that in advanced-stage non-small-cell lung cancer, the proportion of CMTM6-high/PD-L1+cells is low, and some cases with PD-L1 - contain high expression of CMTM6 [18]. CMTM6 was more prevalent than PD-L1 and CMTM6 overexpression was common [19]. Similarly, in our results, we observed that the percentage of CMTM6high/PD-L1+cells was only $36.7 \%$ (116/316) in the MTM type and $31.0 \%(94 / 303)$ in the non-MTM type. To the best of our knowledge, our study is the first to investigate the clinical significance of CMTM6 and PD-L1 coexpression in HCC. We found that CMTM6/PD-L1 can be regarded as a predictor of OS in HCC patients, especially in patients

Fig. 3 PD-L1 and CMTM6 expression in HCC tissue. a Representative micrographs of CMTM6-high and -low, tumoral PD-L1-positive and -negative, inflammatory cell PD-L1-positive and -negative expression. b Scatter plot correlation between CMTM6 and tumoral PD-L1 expression in whole population, MTM type and non-MTM type. c Scatter plot correlation between CMTM6 and inflammatory cell PD-L1 expression in whole population, MTM type and non-MTM type. d Dot plots of inflammatory cell density in different subgroups of whole population. Group I: CMTM6Low/PD-L1-; Group II: CMTM6High/PD-L1 - or CMTM6Low/PD-L1+; Group III: CMTM6High/PD-L1+. Quantitative data are presented as mean \pm SD. $P D-L 1-$ PD-L1-negative, $P D-L 1+$ PD-L1-positive 
A
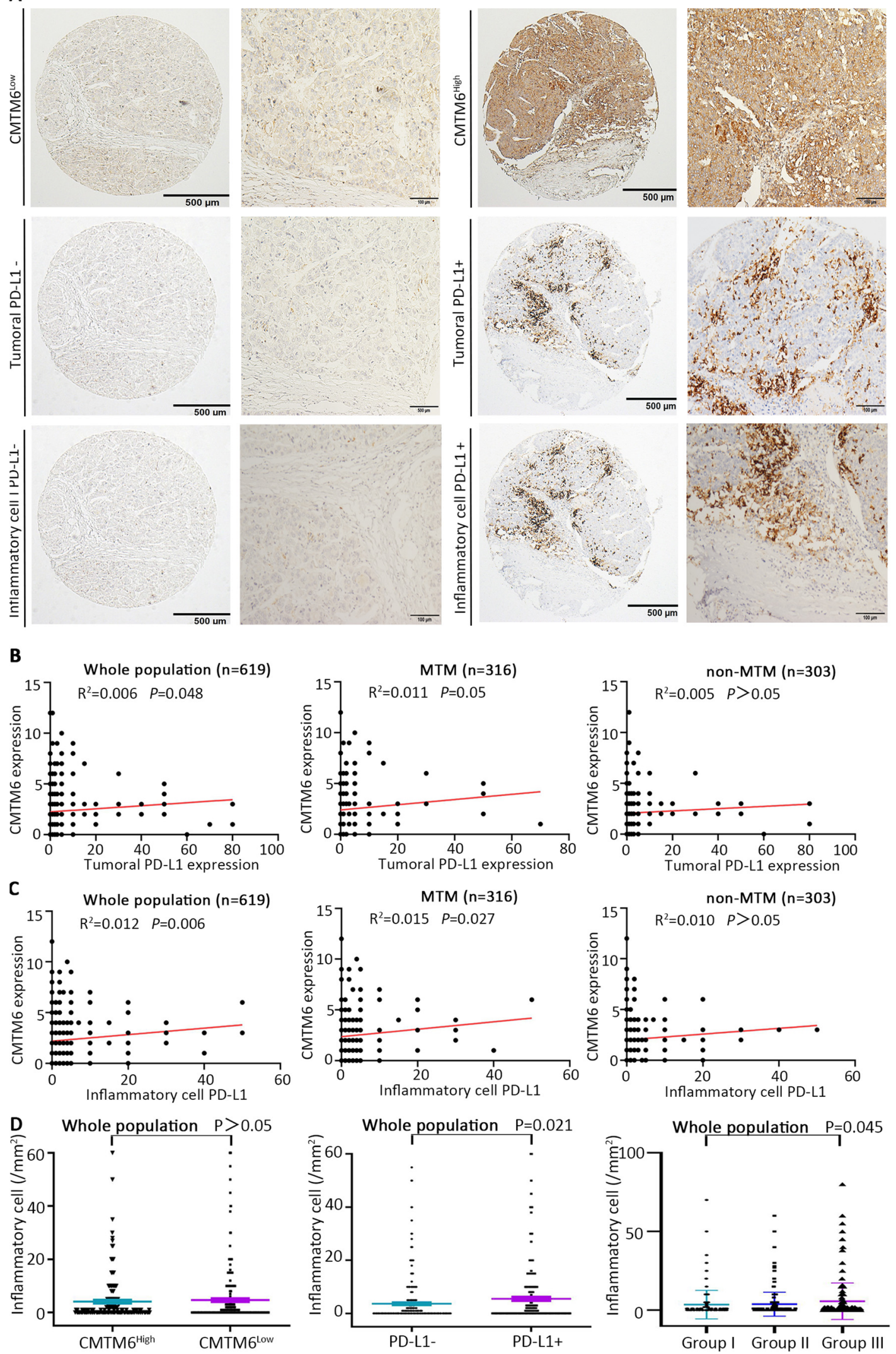
with the MTM subtype. Our study provides some useful insights for immunotherapy of MTM subtypes: patients with CMTM6-high/PD-L1+ status may benefit from the dual blockade of PD-L1 and CMTM6; patients with one positive immune marker (PD-L1 or CMTM6) may need corresponding immune blockage to improve efficacy; for patients with no positive immune maker, immunotherapy targeting other markers, such as PD-L2 or CMTM4, may be needed.

The relationship between CMTM6 expression and inflammatory cell infiltration is controversial. Chen et al. found that high expression of CMTM6 was associated with low inflammatory density in head and neck squamous cell carcinoma [15]. Wang et al. observed that there was a significant correlation between CMTM6 expression and inflammatory cells in lung adenocarcinoma [32]. In our study, we observed that high CMTM6 expression was significantly associated with a high density of inflammatory cells in HCC. We hypothesize that CMTM6 may be induced by inflammatory cells. In addition, we found high inflammatory cell density in the MTM subtype. This result suggests that MTM-type patients are suitable candidates for tumor immunotherapy. In contrast, low levels of inflammatory cell density in the non-MTM subtype suggest that this subtype has features of nonimmunogenic "cold" tumors, in accordance with previous reports on the poor immunogenicity of HCC, as defined by the lack of tumor-infiltrating lymphocytes and a poor response to immunotherapy [33]. According to Teng's theory, it may be much more rational to classify by inflammatory cells via the combination PD-L1 and CMTM6 or in combination with other CMTM family members in HCC [34]. Consistent with previous study, our results demonstrated that in inflammatory cell-positive groups, CMTM6/ PD-L1 coexpression displayed significant prognostic value for DFS and OS in MTM subtype [35]. These results suggested that both CMTM6 and PD-L1 perform immunosuppressive functions that partly depend on suppression of the inflammatory cell function.

There are some limitations to our study. First, due to the retrospective nature of our study, some bias was inevitable. Second, we only detected CMTM6 and PD-L1 to conduct immune classification. Other immunosuppressive factors, such as PD-L2, may also contribute to immunosuppression in the tumor microenvironment. Therefore, an immunophenotyping system containing other markers is needed to guide immunotherapy.

Table 2 Univariate analyses of prognostic factors correlated with OS in MTM and non-MTM HCC

\begin{tabular}{|c|c|c|c|c|c|c|}
\hline \multirow[t]{2}{*}{ Variables } & \multicolumn{2}{|l|}{ Overall } & \multicolumn{2}{|l|}{ MTM } & \multicolumn{2}{|l|}{ Non-MTM } \\
\hline & $\mathrm{HR}(95 \% \mathrm{CI})$ & $P$ value & HR $(95 \% \mathrm{CI})$ & $P$ value & $\mathrm{HR}(95 \% \mathrm{CI})$ & $P$ value \\
\hline \multicolumn{7}{|l|}{ Overall survival } \\
\hline Age $(\text { years })^{\mathrm{a}}(<50$ vs. $\geq 50)$ & $0.886(0.746-1.053)$ & 0.169 & $0.828(0.643-1.067)$ & 0.144 & $0.833(0.584-1.189)$ & 0.315 \\
\hline Gender (male vs. female) & $0.767(0.578-1.017)$ & 0.066 & $0.625(0.391-1.000)$ & $\mathbf{0 . 0 5 0}$ & $1.006(0.794-1.276)$ & 0.958 \\
\hline HBV (positive vs. negative) & $1.331(0.948-1.869)$ & 0.099 & $1.214(0.741-1.991)$ & 0.441 & $1.471(0.922-2.347)$ & 0.106 \\
\hline $\operatorname{AFP}(\mathrm{ng} / \mathrm{ml})(<20 \mathrm{vs} . \geq 20)$ & $1.227(1.005-1.498)$ & 0.045 & $1.263(0.959-1.664)$ & 0.097 & $1.073(0.799-1.441)$ & 0.639 \\
\hline Cirrhosis (no vs. yes) & $1.064(0.841-1.346)$ & 0.604 & $1.110(0.791-1.558)$ & 0.545 & $1.003(0.724-1.390)$ & 0.985 \\
\hline Tumor multiplicity (single vs. multiple) & $1.633(1.359-1.963)$ & 0.000 & $1.771(1.349-2.324)$ & 0.000 & $1.478(1.151-1.897)$ & 0.002 \\
\hline Tumor size $^{\mathrm{b}}(\mathrm{cm})(<5$ vs. $\geq 5)$ & $1.618(1.322-1.980)$ & 0.000 & $1.497(1.136-1.973)$ & 0.004 & $1.665(1.232-2.250)$ & 0.001 \\
\hline Differentiation (well vs. moderate-poor) & $1.535(1.143-2.062)$ & 0.004 & $1.479(1.007-2.170)$ & 0.046 & $1.513(0.949-2.415)$ & 0.082 \\
\hline TNM stage (I-II vs. III-IV) & $1.778(1.491-2.120)$ & 0.000 & $1.874(1.446-2.429)$ & 0.000 & $1.640(1.290-2.086)$ & 0.000 \\
\hline Vascular invasion (no vs. yes) & $2.452(1.952-3.081)$ & 0.000 & $3.472(2.362-5.103)$ & 0.000 & $1.901(1.427-2.532)$ & 0.000 \\
\hline Tumor capsule (complete vs. incomplete) & $0.746(0.627-0.889)$ & 0.001 & $0.657(0.510-0.846)$ & 0.001 & $0.886(0.696-1.130)$ & 0.330 \\
\hline LN metastasis (no vs. yes) & $2.619(1.817-3.775)$ & 0.000 & $4.290(2.524-7.292)$ & 0.000 & $1.754(1.056-2.912)$ & $\mathbf{0 . 0 3 0}$ \\
\hline $\begin{array}{l}\text { Cytological type (liver cell vs. clear cell } \\
\text { vs. fatty-rich vs. giant cell) }\end{array}$ & $0.983(0.860-1.124)$ & 0.799 & $1.062(0.877-1.285)$ & 0.538 & $0.897(0.733-1.096)$ & 0.288 \\
\hline Tumoral PD-L1 (- vs. +) & $1.217(1.021-1.452)$ & 0.029 & $1.610(1.247-2.102)$ & 0.000 & $0.899(0.706-1.144)$ & 0.386 \\
\hline Inflammatory cell PD-L1 (- vs. +) & $1.161(0.978-1.379)$ & 0.089 & $1.458(1.135-1.875)$ & 0.003 & $0.926(0.729-1.175)$ & 0.526 \\
\hline Inflammatory cells (low vs. high) & $1.038(0.872-1.235)$ & 0.676 & $1.198(0.931-1.542)$ & 0.160 & $0.855(0.671-1.089)$ & 0.206 \\
\hline CMTM6 expression (low vs. high) & $1.493(1.250-1.783)$ & 0.000 & $1.574(1.221-2.028)$ & 0.000 & $1.310(1.019-1.684)$ & 0.035 \\
\hline
\end{tabular}

$A F P$ a-fetoprotein, $H B V$ hepatitis B virus infection, $L N$ lymph node, $H R$ hazard ratio, $C I$ confidence interval

* $P$ value $<0.05$ in bold are statistically significant

${ }^{\mathrm{a}}$ Median age; ${ }^{\mathrm{b}}$ Median tumor size 

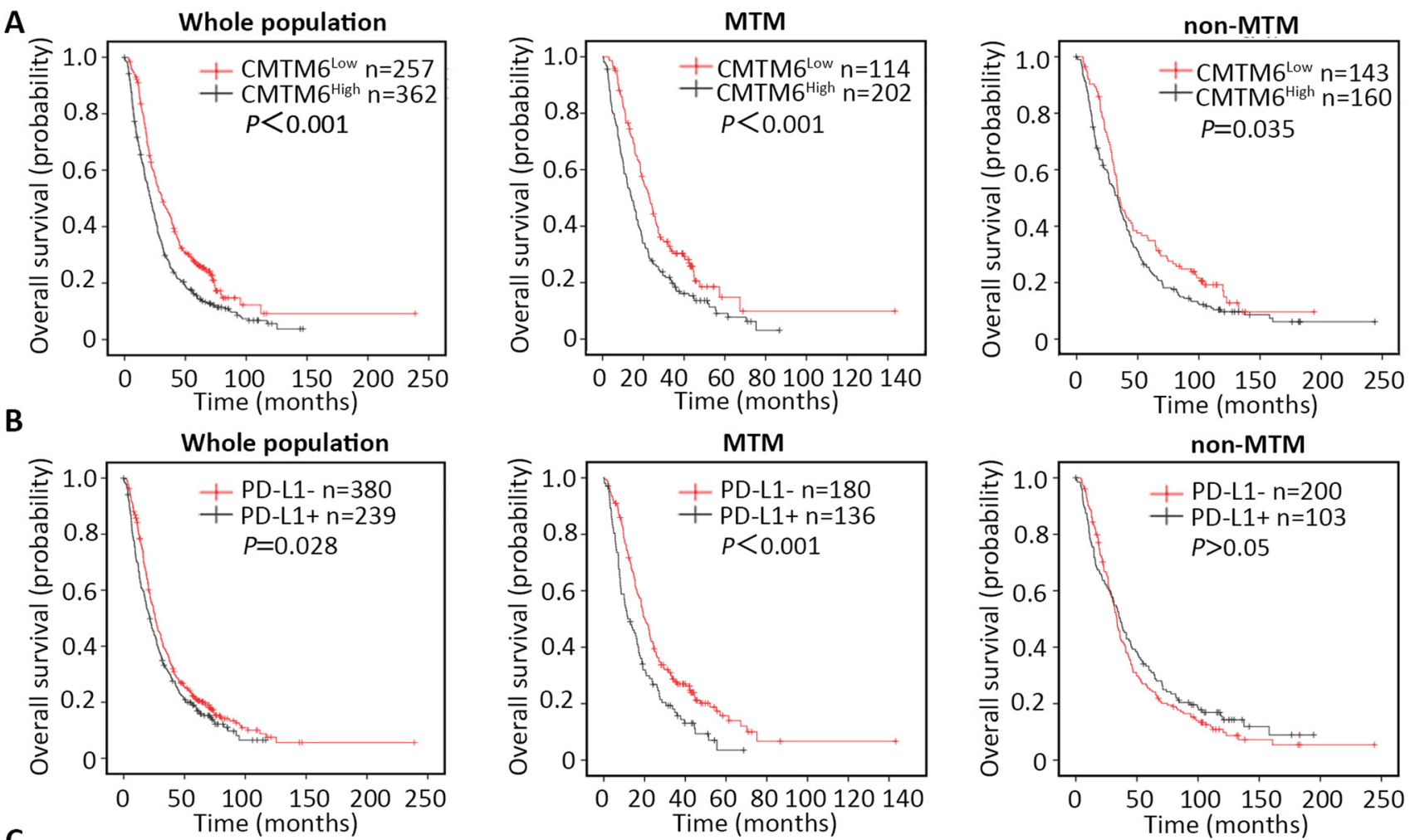

C

Whole population

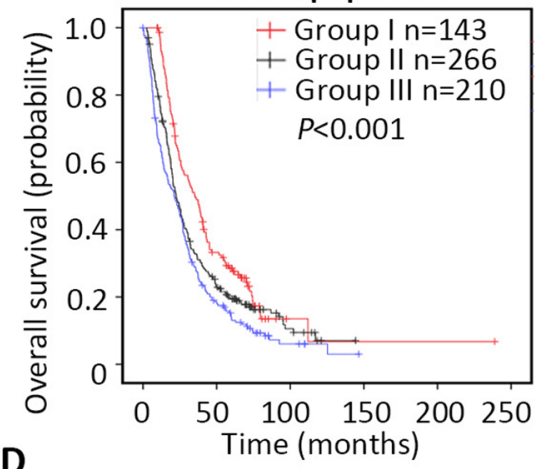

MTM

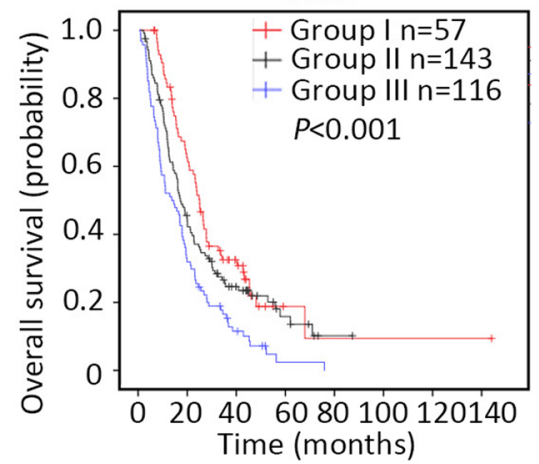

non-MTM
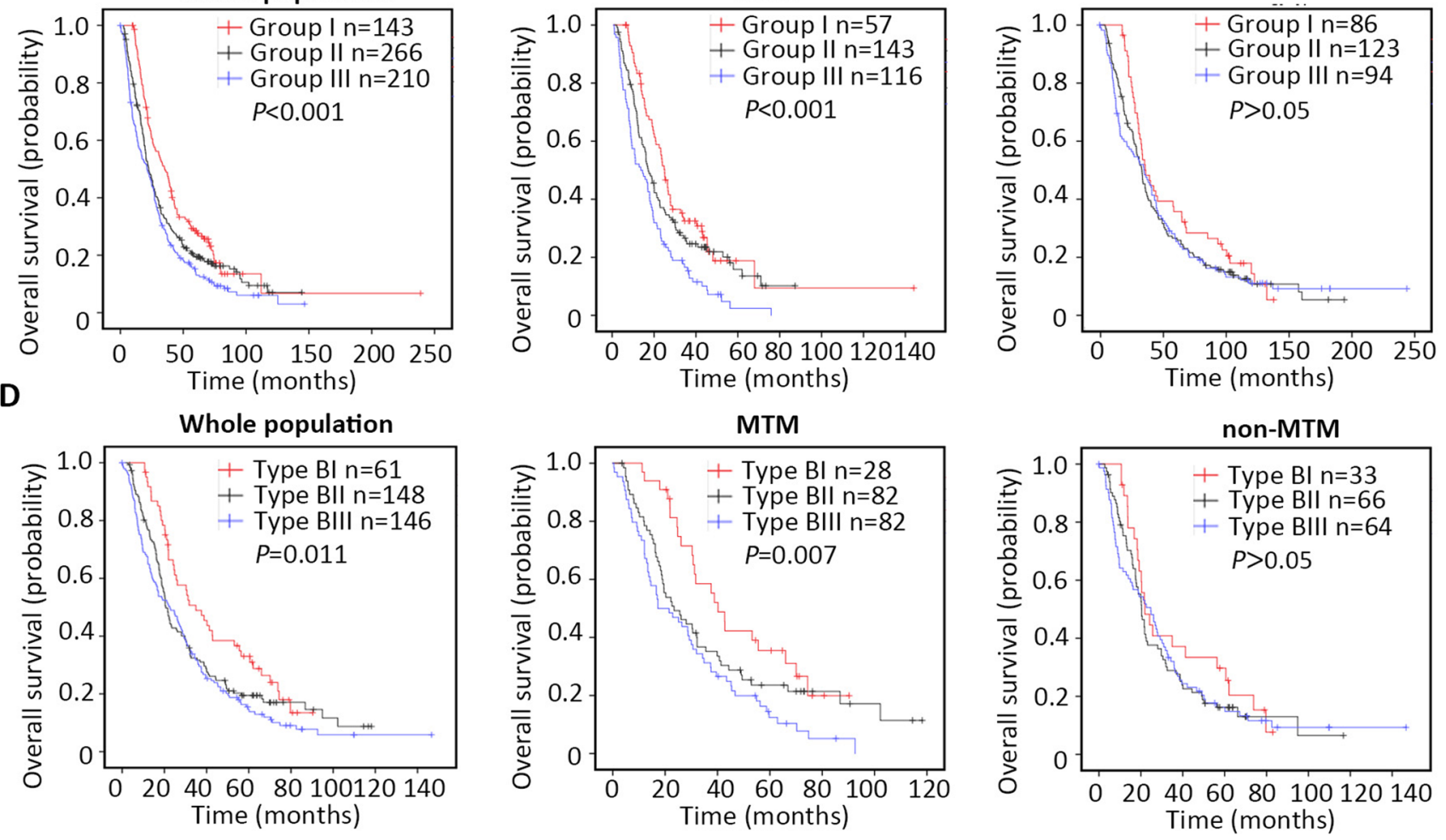

Fig. 4 Kaplan-Meier survival curves for OS of HCC patients according to CMTM6 and PD-L1 expression. a OS according to CMTM6 expression status in the whole population, MTM and non-MTM type HCC. b OS according to tumoral PD-L1 expression status in the whole population, MTM and non-MTM type HCC. c OS according to a combination of CMTM6/PDL1 coexpression. Group I: CMTM6Low/PD-L1-; Group II: CMTM6High/PD-L1- or CMTM6Low/
PD-L1+; Group III: CMTM6High/PD-L1+. d OS according to new immune classification. Type BI: inflammatory cells positive and both negative (CMTM6Low/PD-L1-); type BII: inflammatory cells positive and single positive (CMTM6High/PD-L1- or CMTM6Low/ PD-L1+); type BIII: inflammatory cells positive and both positive (CMTM6High/PD-L1+). PD-L1- PD-L1-negative, $P D-L 1+$ PDL1-positive 
Table 3 Multivariate analyses of prognostic factors correlated with OS in MTM and non-MTM HCC

\begin{tabular}{|c|c|c|c|c|c|c|}
\hline \multirow[t]{2}{*}{ Variables } & \multicolumn{2}{|l|}{ Whole population } & \multicolumn{2}{|l|}{ MTM } & \multicolumn{2}{|l|}{ Non-MTM } \\
\hline & HR (95\% CI) & $P$ value & HR $(95 \%$ CI $)$ & $P$ value & HR $(95 \% \mathrm{CI})$ & $P$ value \\
\hline \multicolumn{7}{|l|}{ Overall survival } \\
\hline Gender (male vs. female) & & & $0.616(0.379-1.000)$ & 0.050 & & \\
\hline $\operatorname{AFP}(\mathrm{ng} / \mathrm{ml})(<20 \mathrm{vs} . \geq 20)$ & $1.016(0.825-1.251)$ & 0.883 & & & & \\
\hline Tumor multiplicity (single vs. multiple) & $1.361(1.110-1.669)$ & 0.003 & $1.672(1.232-2.269)$ & 0.001 & $1.197(0.906-1.582)$ & 0.205 \\
\hline Tumor size $(\mathrm{cm})(<5$ vs. $\geq 5)$ & $1.414(1.127-1.773)$ & 0.003 & $1.427(1.020-1.996)$ & $\mathbf{0 . 0 3 8}$ & $1.446(1.041-2.008)$ & 0.028 \\
\hline Differentiation (well vs. moderate-poor) & $1.275(0.939-1.730)$ & 0.119 & $1.298(0.868-1.941)$ & 0.204 & & \\
\hline TNM stage (I-II vs. III-IV) & $1.114(0.888-1.398)$ & 0.350 & $1.061(0.743-1.514)$ & 0.747 & $1.163(0.861-1.572)$ & 0.325 \\
\hline Vascular invasion (no vs. yes) & $1.742(1.347-2.253)$ & 0.000 & $2.246(1.452-3.474)$ & 0.000 & $1.557(1.130-2.128)$ & 0.005 \\
\hline Tumor capsule (complete vs. incomplete) & $0.916(0.759-1.105)$ & 0.359 & $0.785(0.597-1.032)$ & 0.083 & & \\
\hline LN metastasis (no vs. yes) & $2.032(1.384-2.984)$ & $\mathbf{0 . 0 0 0}$ & $4.049(2.328-7.044)$ & 0.000 & $1.446(0.827-2.425)$ & 0.205 \\
\hline Tumoral PD-L1 (- vs. +) & $1.167(0.973-1.400)$ & 0.097 & $1.384(0.994-1.928)$ & 0.054 & & \\
\hline Inflammatory cell PD-L1 (- vs. +) & & & $1.384(0.883-1.676)$ & 0.231 & & \\
\hline CMTM6 expression (low vs. high) & $1.056(1.005-1.109)$ & 0.030 & $1.116(0.850-1.464)$ & 0.430 & $1.280(0.994-1.647)$ & 0.055 \\
\hline
\end{tabular}

$A F P$ a-fetoprotein, $H B V$ hepatitis B virus infection, $L N$ lymph node, $H R$ hazard ratio, $C I$ confidence interval

$* P$ value $<0.05$ in bold are statistically significant

${ }^{\mathrm{a}}$ Median age; ${ }^{\mathrm{b}}$ Median tumor size

Author contributions Conception and design: JPY and RZL. Performing experiments: LLL and SWZ. Drafting of the article: LLL and XC. Acquisition and interpretation of data, review, editing and approval of the manuscript: all authors.

Funding The National Natural Science Foundation of China (No. $81872012,81872266)$. The National Natural Science Foundation of Guangdong province (No. 2018B030311005) and Science and Technology Program of Guangzhou (201707020038). The authenticity of this article has been validated by uploading the key raw data onto the Research Data Deposit public platform (https://www.researchdata.org. cn) with the approval RDD number RDDB2020001505.

\section{Compliance with ethical standards}

Conflict of interest The authors declare that they have no conflict of interest.

Open Access This article is licensed under a Creative Commons Attribution 4.0 International License, which permits use, sharing, adaptation, distribution and reproduction in any medium or format, as long as you give appropriate credit to the original author(s) and the source, provide a link to the Creative Commons licence, and indicate if changes were made. The images or other third party material in this article are included in the article's Creative Commons licence, unless indicated otherwise in a credit line to the material. If material is not included in the article's Creative Commons licence and your intended use is not permitted by statutory regulation or exceeds the permitted use, you will need to obtain permission directly from the copyright holder. To view a copy of this licence, visit http://creativecommons.org/licenses/by/4.0/.

\section{References}

1. Lozano R, Naghavi M, Foreman K et al (2012) Global and regional mortality from 235 causes of death for 20 age groups in 1990 and 2010: a systematic analysis for the Global Burden of Disease Study 2010. Lancet 380:2095-2128. https://doi. org/10.1016/S0140-6736(12)61728-0

2. Zhou J, Sun HC, Wang Z et al (2018) Guidelines for diagnosis and treatment of primary liver cancer in China (2017 edition). Liver Cancer 7:235-260. https://doi.org/10.1159/000488035

3. Papatheodoridis GV, Sypsa V, Dalekos GN et al (2020) Hepatocellular carcinoma prediction beyond year 5 of oral therapy in a large cohort of Caucasian patients with chronic hepatitis B. J Hepatol. https://doi.org/10.1016/j.jhep.2020.01.007

4. Yang JD, Hainaut P, Gores GJ, Amadou A, Plymoth A, Roberts LR (2019) A global view of hepatocellular carcinoma: trends, risk, prevention and management. Nat Rev Gastroenterol Hepatol 16:589-604. https://doi.org/10.1038/s41575-019-0186-y

5. Calderaro J, Couchy G, Imbeaud S et al (2017) Histological subtypes of hepatocellular carcinoma are related to gene mutations and molecular tumour classification. J Hepatol 67:727-738. https ://doi.org/10.1016/j.jhep.2017.05.014

6. Ziol M, Pote N, Amaddeo G et al (2018) Macrotrabecular-massive hepatocellular carcinoma: a distinctive histological subtype with clinical relevance. Hepatology 68:103-112. https://doi. org/10.1002/hep.29762

7. El-Khoueiry AB, Sangro B, Yau T et al (2017) Nivolumab in patients with advanced hepatocellular carcinoma (CheckMate 040): an open-label, non-comparative, phase $1 / 2$ dose escalation and expansion trial. Lancet 389:2492-2502. https://doi. org/10.1016/S0140-6736(17)31046-2

8. Gao Q, Wang XY, Qiu SJ et al (2009) Overexpression of PD-L1 significantly associates with tumor aggressiveness and postoperative recurrence in human hepatocellular carcinoma. Clin Cancer Res 15:971-979. https://doi.org/10.1158/1078-0432. CCR-08-1608

9. Zhu Y, Yang J, Xu D et al (2019) Disruption of tumour-associated macrophage trafficking by the osteopontin-induced colony-stimulating factor-1 signalling sensitises hepatocellular carcinoma to anti-PD-L1 blockade. Gut 68:1653-1666. https://doi.org/10.1136/ gutjnl-2019-318419 
10. Pinato DJ, Mauri FA, Spina $P$ et al (2019) Clinical implications of heterogeneity in PD-L1 immunohistochemical detection in hepatocellular carcinoma: the Blueprint-HCC study. Br J Cancer 120:1033-1036. https://doi.org/10.1038/s41416-019-0466-x

11. Han W, Ding P, Xu M et al (2003) Identification of eight genes encoding chemokine-like factor superfamily members 1-8 (CKLFSF1-8) by in silico cloning and experimental validation. Genomics 81:609-617. https://doi.org/10.1016/s0888 -7543(03)00095-8

12. Guan X, Zhang C, Zhao J, Sun G, Song Q, Jia W (2018) CMTM6 overexpression is associated with molecular and clinical characteristics of malignancy and predicts poor prognosis in gliomas. EBioMedicine 35:233-243. https://doi.org/10.1016/j.ebiom .2018.08.012

13. Burr ML, Sparbier CE, Chan YC et al (2017) CMTM6 maintains the expression of PD-L1 and regulates anti-tumour immunity. Nature 549:101-105. https://doi.org/10.1038/nature23643

14. Mezzadra R, Sun C, Jae LT et al (2017) Identification of CMTM6 and CMTM4 as PD-L1 protein regulators. Nature 549:106-110. https://doi.org/10.1038/nature23669

15. Chen L, Yang QC, Li YC et al (2020) Targeting CMTM6 suppresses stem cell-like properties and enhances antitumor immunity in head and neck squamous cell carcinoma. Cancer Immunol Res 8:179-191. https://doi.org/10.1158/2326-6066.CIR-19-0394

16. Zhao W, Zhao F, Yang K et al (2019) An immunophenotyping of renal clear cell carcinoma with characteristics and a potential therapeutic target for patients insensitive to immune checkpoint blockade. J Cell Biochem 120:13330-13341. https://doi. org/10.1002/jcb.28607

17. Mamessier E, Birnbaum DJ, Finetti P, Birnbaum D, Bertucci F (2018) CMTM6 stabilizes PD-L1 expression and refines its prognostic value in tumors. Ann Transl Med 6:54. https://doi. org/10.21037/atm.2017.11.26

18. Zugazagoitia J, Liu Y, Toki M et al (2019) Quantitative assessment of CMTM6 in the tumor microenvironment and association with response to PD-1 pathway blockade in advanced-stage nonsmall cell lung cancer. J Thorac Oncol 14:2084-2096. https://doi. org/10.1016/j.jtho.2019.09.014

19. Gao F, Chen J, Wang J, Li P, Wu S, Wang J, Ji Y (2019) CMTM6, the newly identified PD-L1 regulator, correlates with PD-L1 expression in lung cancers. Biochem Biophys Rep 20:100690. https://doi.org/10.1016/j.bbrep.2019.100690

20. Koh YW, Han JH, Haam S, Jung J, Lee HW (2019) Increased CMTM6 can predict the clinical response to PD-1 inhibitors in non-small cell lung cancer patients. Oncoimmunology 8:e1629261. https://doi.org/10.1080/2162402X.2019.1629261

21. Zhu X, Qi G, Li C et al (2019) Expression and clinical significance of CMTM6 in hepatocellular carcinoma. DNA Cell Biol 38:193-197. https://doi.org/10.1089/dna.2018.4513

22. Bei C, Zhang Y, Wei R, Zhu X, Wang Z, Zeng W, Chen Q, Tan $S$ (2017) Clinical significance of CMTM4 expression in hepatocellular carcinoma. OncoTargets Ther 10:5439-5443. https://doi. org/10.2147/OTT.S149786

23. Bei C, Tan C, Zhu X, Wang Z, Tan S (2018) Association between polymorphisms in CMTM family genes and hepatocellular carcinoma in Guangxi of China. DNA Cell Biol 37:691-696. https:// doi.org/10.1089/dna.2018.4274
24. Yafune A, Kawai M, Itahashi M, Kimura M, Nakane F, Mitsumori K, Shibutani M (2013) Global DNA methylation screening of liver in piperonyl butoxide-treated mice in a two-stage hepatocarcinogenesis model. Toxicol Lett 222:295-302. https://doi. org/10.1016/j.toxlet.2013.08.006

25. Herbst RS, Soria JC, Kowanetz M et al (2014) Predictive correlates of response to the anti-PD-L1 antibody MPDL3280A in cancer patients. Nature 515:563-567. https://doi.org/10.1038/ nature 14011

26. Chen CL, Pan QZ, Zhao JJ et al (2016) PD-L1 expression as a predictive biomarker for cytokine-induced killer cell immunotherapy in patients with hepatocellular carcinoma. Oncoimmunology 5:e1176653. https://doi.org/10.1080/2162402X.2016.1176653

27. Schonrich G, Raftery MJ (2019) The PD-1/PD-L1 axis and virus infections: a delicate balance. Front Cell Infect Microbiol 9:207. https://doi.org/10.3389/fcimb.2019.00207

28. Huang ZY, Xu P, Li JH et al (2017) Clinical significance of dynamics of programmed death ligand-1 expression on circulating CD14(+) monocytes and CD19(+) B cells with the progression of hepatitis B virus infection. Viral Immunol 30:224-231. https ://doi.org/10.1089/vim.2016.0122

29. Kakizaki M, Yamamoto Y, Yabuta S, Kurosaki N, Kagawa T, Kotani A (2018) The immunological function of extracellular vesicles in hepatitis B virus-infected hepatocytes. PLoS One 13:e0205886. https://doi.org/10.1371/journal.pone.0205886

30. Han X, Gu YK, Li SL, Chen H, Chen MS, Cai QQ, Deng HX, Zuo MX, Huang JH (2019) Pre-treatment serum levels of soluble programmed cell death-ligand 1 predict prognosis in patients with hepatitis B-related hepatocellular carcinoma. J Cancer Res Clin Oncol 145:303-312. https://doi.org/10.1007/s00432-018-2758-6

31. Ng CT, Snell LM, Brooks DG, Oldstone MB (2013) Networking at the level of host immunity: immune cell interactions during persistent viral infections. Cell Host Microbe 13:652-664. https ://doi.org/10.1016/j.chom.2013.05.014

32. Wang H, Gao J, Zhang R, Li M, Peng Z, Wang H (2020) Molecular and immune characteristics for lung adenocarcinoma patients with CMTM6 overexpression. Int Immunopharmacol 83:106478. https://doi.org/10.1016/j.intimp.2020.106478

33. Jiang SS, Tang Y, Zhang YJ et al (2015) A phase I clinical trial utilizing autologous tumor-infiltrating lymphocytes in patients with primary hepatocellular carcinoma. Oncotarget 6:4133941349. https://doi.org/10.18632/oncotarget.5463

34. Teng MW, Ngiow SF, Ribas A, Smyth MJ (2015) Classifying cancers based on T-cell infiltration and PD-L1. Cancer Res 75:2139 2145. https://doi.org/10.1158/0008-5472.CAN-15-0255

35. Zhou QH, Li KW, Chen X et al (2020) HHLA2 and PD-L1 coexpression predicts poor prognosis in patients with clear cell renal cell carcinoma. J Immunother Cancer. https://doi.org/10.1136/jitc2019-000157

Publisher's Note Springer Nature remains neutral with regard to jurisdictional claims in published maps and institutional affiliations. 